\title{
Genetics of Resistance in F2 Soybean Populations for Adzuki Bean Bruchid (Callosobruchus chinensis)
}

\author{
Ulemu Mercy Msiska ${ }^{1}$, Mehari Gebremedhn Hailay ${ }^{1}$, Belay Weldekidan Miesho ${ }^{1}$, Angele Pembele Ibanda ${ }^{1}$, \\ Phinehas Tukamuhabwa $^{1}$, Samuel Kyamanywa ${ }^{1}$, Thomas Lapaka Odong ${ }^{1} \&$ Patrick Rubaihayo ${ }^{1}$ \\ ${ }^{1}$ Department of Agricultural Production, College of Agricultural and Environmental Sciences, Makerere \\ University, Kampala, Uganda \\ Correspondence: Ulemu Mercy Msiska, Department of Agricultural Production, College of Agricultural and \\ Environmental Sciences, Makerere University, Kampala, Uganda. Tel: 256-757-668-227. E-mail: \\ ulemsiska@gmail.com
}

Received: August 6, 2018

Accepted: September 11, 2018

Online Published: November 15, 2018

doi:10.5539/jas.v10n12p171

URL: https://doi.org/10.5539/jas.v10n12p171

The research was financed by Malawi Government through APPSA Project, Intra ACP-Mobility Scheme (CSAA) and Carnegie Cooperation of New York through RUFORUM.

\begin{abstract}
Adzuki bean bruchid (Callosobruchus chinensis) is a significant pest of soybean in Uganda. To sustainably manage this pest, utilization of resistant soybean varieties is the key solution. Development of resistant varieties needs knowledge on modes of inheritance which is crucial in selection of parent materials. To identify parents, a study was initiated to determine the gene action and mode of inheritance of resistance to bruchids in soybean. Nine parental lines were crossed in a full-diallel at Makerere University Agricultural Institute, Uganda. The generated $F_{1}$ s were advanced to $F_{2}$ and seeds were evaluated for response to bruchid infestation in a randomised complete block design. Ten seeds were infested with 10 randomly selected unsexed 1-3 day old bruchids. Genotypes showed significant differences in seed weight loss (swl), adult bruchid emergence (ABE) and Dobie susceptibility index (DSI) indicating that these parameters could be used to screen genotypes in genetic analysis. Mean squares of general combining ability $(\mathrm{GCA})$ were significant $(\mathrm{P}<0.05)$ for swl, DSI and number of ABE from the $F_{2}$ seeds indicating additive gene action. Susceptibility parameters ABE and DSI showed significant specific combining ability (SCA) indicating non-additive gene action. Resistance was influenced by maternal effects indicating that direction of the cross was important. Genotypes S-Line 9.2 and S-Line 13.2A showed negative significant GCA effects for at least two of the susceptibility parameters indicating that they were the best parents for bruchid resistance breeding. The study established that additive, non additive and maternal effects governed the gene expression in soybean resistance to bruchids.
\end{abstract}

Keywords: inheritance, maternal effect, seed weight loss, bruchids, DSI

\section{Introduction}

Adzuki bean bruchid (Callosobruchus chinensis) is a major storage threat to soybean in Uganda. The pest is cosmopolitan and its damage is irreversible. Losses of up to $20 \%$ have been reported in other legumes (Qazi, 2007). Although this pest was reported on pigeon peas in the 1990s (Nahdy, 1995), it was not a known pest of soybean until recently as reported by Tukamuhabwa (2015, personal communication). Consequently there has been no genetic research work done in soybeans related to $C$. chinensis. Genetic resistance is the most economic and environmentally friendly strategy in pest management (Dent, 2000). To develop an efficient and successful resistance breeding programme, understanding the gene actions controlling resistance is fundamental. Inheritance studies of resistance to storage pests have previously been conducted in other legumes (Somta et al., 2007) and cereal crops (Zunjare et al., 2015), however information from the literature on bruchid resistance inheritance studies in soybean is scanty. The genetic control of resistance to storage insect pests may range from monogenic to polygenic (Dent, 2000; Singh, 2009). Mostly additive and dominant genes may govern storage insect pest resistance in many legumes but a few cases of cytoplasmic gene effect have also been reported (Singh, 2009; Keneni et al., 2011). 
Somta et al. (2007) reported that inheritance of resistance to bruchids was complicated. Adjadi et al. (1985) working on cowpea reported high maternal effects, digenic inheritance and complete dominance of susceptibility indicating that resistance was controlled by two recessive genes. However in a cross between mungbean and blackgram, Dongre et al. (1996) found that resistance to C. maculatus was controlled by two dominant duplicate genes. Kananji (2007) reported that although both additive and dominance gene action governed the expression of resistance, the dominance component was relatively more important for the bean bruchids. Kananji (2007) also reported low heritability in the narrow sense, suggesting that breeding for bruchid resistance cannot be easy.

Based on phenotypic performance to bruchids resistance alone, may not provide adequate information to guide selection of elite parents and desirable cross combination required for systematic breeding hence the need for combining ability studies. Combining ability or productivity in crosses is defined as the cultivars or parents ability to combine among each other during hybridization process such that desirable genes or characters are transmitted to their progenies (Fasahat et al., 2016). Combining abilities are categorized into two as general (GCA) and specific (SCA) combining abilities. GCA represents the activity of genes which are largely additive in their effects while SCA is regarded as an indication of loci with dominance variance and/or epistasis (Fasahat et al., 2016). It was therefore imperative to carry out a study with the objectives of determining the mode of gene action and inheritance of resistance to $C$. chinensis in soybean.

\section{Materials and Methods}

\subsection{Experimental Site and Germplasm}

Nine soybean lines Maksoy 1N, Maksoy 3N, Maksoy 4N, AVRDC G8527, G7955, S-Line 13.2A, S-Line 9.2, SREB-15C and UG 5 (Table 1) with varying levels of resistance were crossed in a screen house at Makerere University Agricultural Research Institute-Kabanyolo (MUARIK) in the year 2016 using full diallel mating design to produce F1s (Plate 1) (Choudhary et al., 2004). The soybean lines were previously characterized for bruchid resistance at MUARIK (Msiska et al., 2018). Soybean seed was provided by the Soybean Breeding and Seed Systems program of Makerere University. $F_{1}$ seeds were grown in pots together with their corresponding female parent in the screen house to generate adequate $F_{2}$ seeds (Maphosa, 2013).
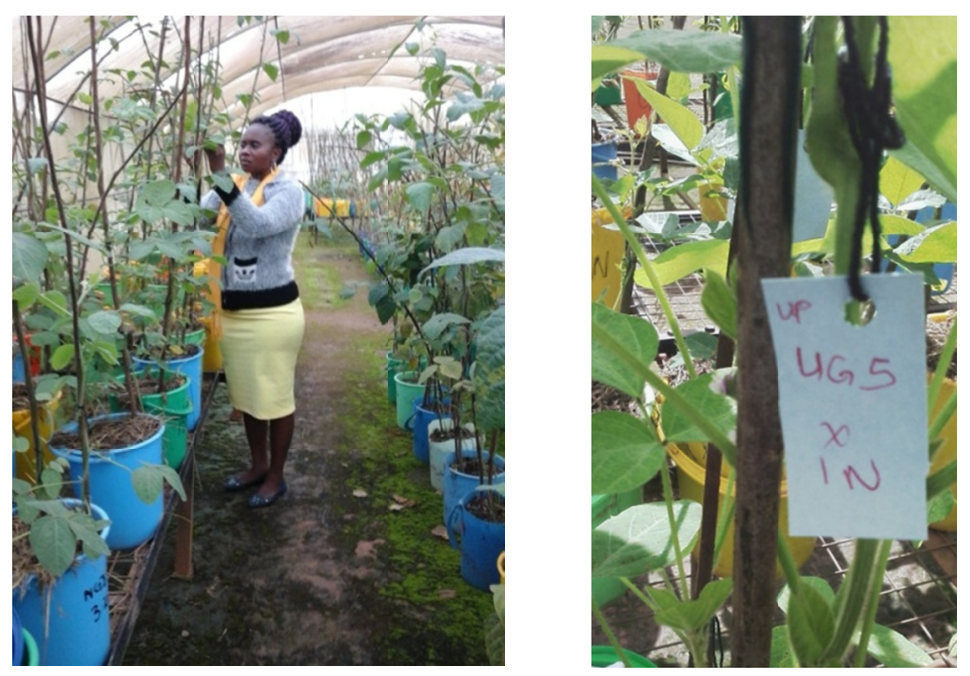

Plate 1. Soybean crossing in the screen-house at MUARIK 2016

\subsection{Data Collection}

The parents and $\mathrm{F}_{2}$ seeds were evaluated for resistance to $C$. chinensis in a laboratory in a randomized complete block design (RCBD) with insect infestation days as blocks; and were replicated thrice (Abebe et al., 2009). A total of 10 seeds were weighed and placed in a petridish after 24 hours of oven drying. The seeds were then infested with 10 unsexed 1-3 day old bruchids from an established colony under the no-choice test method as described by Msiska et al. (2018). Prior to the study, insects were cultured on susceptible soybean genotypes Maksoy $2 \mathrm{~N}$ and Maksoy $3 \mathrm{~N}$ so as to eliminate any short term changes in behavior associated with the change of host plant from that used for culturing to that being tested (Seram et al., 2016). Data on initial seed weights (iwt), 
number of eggs, adult bruchid emergence (ABE), final weights (fwt) were collected. Emerging bruchids were recorded and removed daily for the determination of median development period (Msiska et al., 2018). Subsequently seed weight loss (swl), percentage seed weight loss (swl\%), percentage adult insect emergence (\%IE), growth index (GI), median development period (MDP), and susceptibility index (DSI) were calculated.

Table 1. Parental materials used in the study during 2016/2017

\begin{tabular}{lllll}
\hline ID & Name & Pedigree & Source & Susceptibility status \\
\hline 1 & Maksoy 1N & TGX 1835-10E & Uganda & Moderate Resistant \\
2 & Maksoy 3N & GC 00138-29 $\times$ Duiker & Uganda & Susceptible \\
3 & Maksoy 4N & Duiker $\times$ GC 00138-29 & Uganda & Susceptible \\
4 & G7955 & Unspecified $\times$ Unspecified & Taiwan & Resistant \\
5 & AVRDC G8527 & Unspecified $\times$ Unspecified & Taiwan & Resistant \\
6 & S-Line 13.2A & Unspecified $\times$ Unspecified & Uganda & Moderate Resistant \\
7 & S-Line 9.2 & Unspecified $\times$ Unspecified & Uganda & Moderate Resistant \\
8 & SREB-15C & Unspecified $\times$ Unspecified & Taiwan & Moderate Resistant \\
9 & UG 5 & Unspecified $\times$ Unspecified & Uganda & Very Susceptible \\
\hline
\end{tabular}

Note. Source: Msiska et al. 2018.

(1) Seed weight loss \%, which is an economic loss indicator (Amusa et al., 2014), was calculated as follows:

$$
\text { Seed weight loss } \%=\frac{100 \times(i w t-f w t)}{i w t}
$$

where, $i w t=$ Initial seed weight, $f w t=$ Final Seed weight for the sample.

(2) Growth Index (GI), which is an indicator of genotype suitability for development of insects (Wijenayake \& Karunaratne, 1999) was calculated as:

$$
\text { Growth Index }(\mathrm{GI})=\frac{\% A B E}{M D P}
$$

The median development period (MDP) was calculated as the number of days from the middle of oviposition (d 5) to the first progeny emergence (Kananji, 2007).

(3) Dobie Susceptibility Index (DSI): The data on the number of adult bruchid that emerged and the median development period were used to calculate the Dobie susceptibility index (Dobie, 1974) for each genotype using the formula:

$$
\text { Dobie Susceptibility Index (DSI) }=\frac{\log _{e} Y \times 100}{t}
$$

where, $Y=$ total number of adult bruchid emerged and $t=$ median development period.

If no insect emerged over the test period, the Dobie susceptibility index value was equal to zero (DSI $=0$ ) (Derera et al., 2001). The modified Dobie (1974) susceptibility index ranging from 0-9 was used to classify the soybean genotypes; where, $0-1=$ resistant; $2-3=$ moderate resistant; $4-5=$ susceptible and $\geq 6$ highly susceptible (Radha \& Susheela, 2014). The genotypes with a high susceptibility index (DSI) were considered susceptible and those with a low susceptibility index as resistant (Msiska et al., 2018). This was based on the assumption that a few insect progenies would emerge out of a resistant genotype and insect progeny development would take a longer time in a resistant than in a susceptible genotype (Kananji, 2007)

\subsection{Data Analysis}

The assumptions of ANOVA were tested before data analysis using GenStat $12^{\text {th }}$ Edition procedures. Data on GI and MDP were transformed using Log (base10) function. Data were then subjected to one-way analysis of variance with genotypes as treatment factor while replication as blocks using GenStat $12^{\text {th }}$ Edition statistical package (Harding \& Payne, 2012) following linear statistical model:

$$
\gamma_{i j}=\mu+\tau_{i}+\beta_{j}+\varepsilon_{i j}
$$

Where, $\gamma_{i j}=$ observed value for the $i^{\text {th }}$ genotype in $j^{\text {th }}$ block, $\mu=$ Overall mean effect, $\tau_{i}=$ Genotype effect (fixed), $\beta_{j}=j^{\text {th }}$ block effect (random), $\varepsilon_{i j}=$ Error term.

Means of parameters which were significantly different from the ANOVA were analysed using analysis of genetic designs (AGD-R) statistical package (Rodriguez et al., 2015) to generate variance components, GCA, 
SCA and maternal effects. The statistical model for analysis was based on Griffing (1956) method 1 model 1 as described by Hallauer et al. (1988):

$$
X_{i j k}=\mu+r_{k}+g_{i}+g_{j}+s_{i j}+m_{i j}+p_{i j k}
$$

where, $X_{i j k}$ is the value of the progeny derived from the crossing of the ith female parent with jth male parent, $u$ is the mean effect for all progenies, $r_{k}$ is the replication effect, $g_{i}$ is the GCA effects of the ith female parent, $g_{j}$ are the GCA effects of the jth male parent, $s_{i j}$ is the SCA effect specific to the hybrid of the $\mathrm{i}$-th female and the $\mathrm{j}$-th male genotype, $m i j$ is reciprocal effects, $p_{i j k}$ is the experimental error for the $X_{i j k}$ observation $(k=1,2, \ldots 81 ; i$ $=j=1,2, \ldots 9$ ). Grrifing's method 1 model 1 is a numerical approach where genotypes are fixed, includes parents, progenies and reciprocals. Fixed model was used because parental lines were selected purposely, based on their levels of resistance to C. chinensis (Choudhary et al., 2004).

GCA and SCA effects were estimated as described by Hallauer et al. (1988) respectively, as:

$$
\begin{gathered}
\sigma_{i}=\{1 /[n(n-2)]\}\left(n X_{i \cdot}-2 X_{. .}\right) \\
s_{i j}=X_{i j}-[1 /(n-2)]\left(X_{i}+X_{j}\right)+\{2 /[(n-1)(n-2)]\} X_{. .}
\end{gathered}
$$

Significance of GCA, SCA, maternal and reciprocal effects was determined by a $t$ tests as described by Griffing (1956), and Dabholkar (1999). GCA was used to estimate genetic variations in parents. Assuming no additive-by-additive interactions and other higher terms, GCA may be used to estimate heritability for traits. For traits with significant GCA effects, heritability and phenotypic variance were calculated by the formulae that follow respectively:

$$
\begin{aligned}
& \mathrm{h}^{2}=\frac{2 \delta_{\mathrm{GCA}}^{2}}{2 \delta_{\mathrm{GCA}}^{2}+\delta_{\mathrm{e}}^{2}} \\
& \delta_{\mathrm{p}}^{2}=2 \delta_{\mathrm{GCA}}^{2}+\delta_{\mathrm{e}}^{2}
\end{aligned}
$$

Baker's Ratio which estimates the relative significance of additive to non additive gene effects (Mwije et al., 2014) and allows inferences about optimum allocation of resources in hybrid breeding (Fasahat et al., 2016) was calculated using formula:

$$
\text { Baker's Ratio }=\frac{2 \delta_{\mathrm{GCA}}}{2 \delta_{\mathrm{GCA}}+\delta_{\mathrm{SCA}}}
$$

where, $\delta_{\mathrm{GCA}}=$ General combining ability variance, $\delta_{\mathrm{SCA}}=$ specific combining ability variance.

\section{Results}

Mean squares for susceptibility parameters on soybean seed are shown in Table 2. Significant differences were observed among $\mathrm{F}_{2}$ population for swl, $\mathrm{ABE}$ and DSI. No significant differences were observed among genotypes for number of eggs, \%IE, MDP and GI.

Table 3 presents means on response of parents and $\mathrm{F}_{2}$ generation to $C$. chinensis. The most susceptible genotypes were UG $5(\mathrm{DSI}=4.99)$, Maksoy $3 \mathrm{~N}(\mathrm{DSI}=5.96)$ and Maksoy $4 \mathrm{~N}(\mathrm{DSI}=6.08)$. Most resistant genotypes were S-Line $9.2 \times$ Maksoy 3N (DSI $=0.002)$, SREB-15C $\times$ Maksoy 3N $($ DSI $=0.002)$ and SREB-15C $\times$ S-Line 9.2 $(\mathrm{DSI}=0.002)$. Genotypes with the highest ABE were Maksoy $3 \mathrm{~N}$ (adults $=68$ ), UG 5 (adults $=65$ ) and Maksoy $4 \mathrm{~N}$ (adults $=53$ ). The lowest number of ABE was observed in genotype S-Line $9.2 \times$ Maksoy 3N, SREB-15C $\times$ Maksoy $3 \mathrm{~N}$ and SREB-15C $\times$ S-Line 9.2 (adult $=0.32$ ). All crosses exhibited lower DSI values than their female parent except Maksoy IN and S-Line 9.2 crosses. Crosses with Maksoy 1N and S-Line 9.2 as female parent had higher DSI values than their female parent.

Table 2. Analysis of variance for Callosobruchus chinensis susceptibility parameters in $\mathrm{F}_{2}$ generation

\begin{tabular}{llllllllll}
\hline Source & df. & swl & swl\% & Eggs & ABE & \%IE & MDP & GI & DSI \\
\hline Rep & 2 & $0.03^{\text {ns }}$ & $95.6^{\text {ns }}$ & $3194.5^{*}$ & $184.96^{\text {ns }}$ & $101092^{\text {ns }}$ & $239^{\text {ns }}$ & $126.3^{\text {ns }}$ & $9.52^{*}$ \\
Genotype & 78 & $0.05^{* *}$ & $266.8^{* *}$ & $676.3^{\text {ns }}$ & $426.08^{* * *}$ & $123575^{\text {ns }}$ & $218.9^{\text {ns }}$ & $158.4^{\text {ns }}$ & $4.61^{* *}$ \\
Residual & 154 & 0.03 & 163.1 & 701.5 & 90.6 & 91683 & 172.2 & 130.8 & 2.77 \\
Total & 234 & 0.04 & 197 & 714.4 & 203.23 & 102394 & 188.3 & 140 & 3.44 \\
\hline
\end{tabular}

Note. df. $=$ degrees of freedom, $\mathrm{swl}=$ Seed weight loss, $\mathrm{swl} \%=$ Percentage seed weight loss, Eggs $=$ Number of eggs, $\mathrm{ABE}=$ Adult bruchid emergence, $\% \mathrm{IE}=$ Percentage adult insect emergence, $\mathrm{MDP}=$ Median Development Period, GI $=$ Growth index, DSI $=$ Dobie susceptibility index, $\mathrm{ns}=$ not significant, $* * *=\mathrm{P}<0.001, * *=\mathrm{P}<$ $0.01, *=\mathrm{P}<0.05$. 
Table 3. Mean performance of genotypes to Callosobruchus chinensis

\begin{tabular}{|c|c|c|c|c|c|c|c|c|}
\hline Genotype & swl & swl\% & Eggs & ABE & $\%$ IE & GI & MDP & DSI \\
\hline $1 \mathrm{~N}$ & 0.04 & 4.38 & 57.26 & 9.98 & 16.06 & 0.39 & 41.31 & 2.13 \\
\hline $1 \mathrm{~N} \times 3 \mathrm{~N}$ & 0.06 & 7.6 & 28.26 & 12.32 & 46.75 & 1.27 & 36.64 & 2.97 \\
\hline $1 \mathrm{~N} \times 4 \mathrm{~N}$ & 0.05 & 7.21 & 54.93 & 15.32 & 32.32 & 1.02 & 31.98 & 3.8 \\
\hline $1 \mathrm{~N} \times \mathrm{G} 7955$ & 0.07 & 7.36 & 20.59 & 8.98 & 42.46 & 0.52 & 10.31 & 1.48 \\
\hline $1 \mathrm{~N} \times \mathrm{G} 8527$ & 0.03 & 5.7 & 31.26 & 5.65 & 19.75 & 0.52 & 40.31 & 1.36 \\
\hline $1 \mathrm{~N} \times \mathrm{S}$-Line $13.2 \mathrm{~A}$ & 0.07 & 7.22 & 31.59 & 10.32 & 29.49 & 0.76 & 40.64 & 2.26 \\
\hline $1 \mathrm{~N} \times$ S-Line 9.2 & 0.08 & 6.65 & 28.26 & 8.65 & 48.12 & 1.36 & 35.31 & 2.62 \\
\hline $1 \mathrm{~N} \times \mathrm{SREB}-15 \mathrm{C}$ & 0.079 & 6.5 & 33.93 & 12.32 & 36.62 & 0.95 & 24.98 & 2.15 \\
\hline $1 \mathrm{~N} \times \mathrm{UG} 5$ & 0.12 & 11.47 & 52.93 & 21.65 & 35.71 & 1.01 & 36.98 & 2.76 \\
\hline $3 \mathrm{~N}$ & 0.19 & 19.9 & 70.59 & 68.98 & 98.01 & 3.18 & 30.98 & 5.96 \\
\hline $3 \mathrm{~N} \times 1 \mathrm{~N}$ & 0.27 & 24.61 & 58.26 & 17.98 & 52.4 & 1.6 & 33.98 & 3.43 \\
\hline $3 \mathrm{~N} \times 4 \mathrm{~N}$ & 0.21 & 17.78 & 65.59 & 16.98 & 24.15 & 0.69 & 37.64 & 3.21 \\
\hline $3 \mathrm{~N} \times \mathrm{G} 7955$ & 0.22 & 17.73 & 32.93 & 9.65 & 17.28 & 0.49 & 24.64 & 1.73 \\
\hline $3 \mathrm{~N} \times \mathrm{G} 8527$ & 0.23 & 18.38 & 52.93 & 15.98 & 35.96 & 1.07 & 34.64 & 3.12 \\
\hline $3 \mathrm{~N} \times \mathrm{S}$-Line $13.2 \mathrm{~A}$ & 0.13 & 16.56 & 53.59 & 21.65 & 41.14 & 1.13 & 35.98 & 3.55 \\
\hline $3 \mathrm{~N} \times$ S-Line 9.2 & 0.2 & 16.1 & 38.93 & 18.32 & 64.94 & 1.96 & 32.98 & 3.77 \\
\hline $3 \mathrm{~N} \times \mathrm{SREB}-15 \mathrm{C}$ & 0.17 & 18.39 & 23.93 & 8.32 & 27.63 & 0.85 & 21.64 & 2.24 \\
\hline $3 \mathrm{~N} \times \mathrm{UG} 5$ & 0.38 & 34.49 & 21.59 & 13.32 & 66.81 & 0.97 & 23.31 & 2.02 \\
\hline $4 \mathrm{~N}$ & 0.2 & 18.09 & 69.93 & 53.32 & 79.44 & 2.37 & 16.64 & 6.08 \\
\hline $4 \mathrm{~N} \times 1 \mathrm{~N}$ & 0.42 & 32.49 & 12.26 & 5.98 & 19.74 & 0.62 & 10.64 & 1.31 \\
\hline $4 \mathrm{~N} \times 3 \mathrm{~N}$ & 0.2 & 17.4 & 42.26 & 18.32 & 45.32 & 1.37 & 33.31 & 3.74 \\
\hline $4 \mathrm{~N} \times \mathrm{G} 7955$ & 0.17 & 15.72 & 27.59 & 10.65 & 50.56 & 1.41 & 23.98 & 2.17 \\
\hline $4 \mathrm{~N} \times \mathrm{G} 8527$ & 0.23 & 20.87 & 27.59 & 12.65 & 33.57 & 1.25 & 19.31 & 3.38 \\
\hline $4 \mathrm{~N} \times \mathrm{S}$-Line $13.2 \mathrm{~A}$ & 0.18 & 19.37 & 50.26 & 21.98 & 50.52 & 1.59 & 32.31 & 4.07 \\
\hline $4 \mathrm{~N} \times$ S-Line 9.2 & 0.33 & 24.47 & 52.26 & 24.32 & 42.22 & 1.3 & 34.64 & 3.62 \\
\hline $4 \mathrm{~N} \times \mathrm{SREB}-15 \mathrm{C}$ & 0.19 & 15.49 & 24.59 & 8.65 & 18.44 & 0.6 & 10.31 & 1.52 \\
\hline $4 \mathrm{~N} \times \mathrm{UG} 5$ & 0.27 & 20.24 & 47.59 & 21.65 & 66.05 & 1.95 & 33.64 & 3.67 \\
\hline G7955 & 0.11 & 11.38 & 36.26 & 11.32 & 42.42 & 1.17 & 35.98 & 2.5 \\
\hline $\mathrm{G} 7955 \times 1 \mathrm{~N}$ & 0.1 & 11.14 & 39.93 & 5.65 & 15.27 & 0.427 & 23.98 & 1.59 \\
\hline $\mathrm{G} 7955 \times 3 \mathrm{~N}$ & 0.08 & 9.04 & 38.93 & 11.98 & 33.43 & 0.9 & 37.31 & 2.87 \\
\hline $\mathrm{G} 7955 \times 4 \mathrm{~N}$ & 0.26 & 24.21 & 59.59 & 28.32 & 52.39 & 1.5 & 33.98 & 4.16 \\
\hline $\mathrm{G} 7955 \times \mathrm{G} 8527$ & 0.16 & 20.36 & 53.26 & 22.65 & 44.36 & 1.26 & 35.64 & 3.74 \\
\hline G7955 $\times$ S-Line $13.2 \mathrm{~A}$ & 0.12 & 14.54 & 36.93 & 14.98 & 46.38 & 1.31 & 35.64 & 3.13 \\
\hline G7955 × S-Line 9.2 & 0.03 & 2.36 & 38.34 & 5.57 & -3.15 & -0.37 & 46.81 & 1.2 \\
\hline G7955 $\times$ SREB-15C & 0.04 & 4.25 & 46.26 & 21.65 & 48.21 & 1.36 & 35.98 & 3.71 \\
\hline G7955 × UG 5 & 0.1 & 11.83 & 17.28 & 5.02 & 42.02 & 0.95 & 40.89 & 1.8 \\
\hline G8527 & 0.03 & 4.12 & 36.93 & 6.65 & 54.69 & 1.45 & 38.64 & 1.96 \\
\hline $\mathrm{G} 8527 \times 1 \mathrm{~N}$ & 0.01 & 1.94 & 21.59 & 4.32 & 14.27 & 0.39 & 25.31 & 0.97 \\
\hline $\mathrm{G} 8527 \times 3 \mathrm{~N}$ & 0.26 & 36.21 & 39.59 & 10.32 & 34.23 & 0.49 & 34.98 & 2.14 \\
\hline $\mathrm{G} 8527 \times 4 \mathrm{~N}$ & 0.04 & 5.09 & 32.93 & 14.65 & 47.86 & 1.32 & 36.31 & 3.27 \\
\hline $\mathrm{G} 8527 \times \mathrm{G} 7955$ & 0.04 & 3.53 & 61.93 & 10.65 & 26.14 & 0.72 & 36.64 & 2.72 \\
\hline G8527 $\times$ S- Line $13.2 \mathrm{~A}$ & 0.03 & 2.27 & 47.26 & 5.32 & 11.49 & 0.3 & 37.31 & 1.55 \\
\hline G8527 × S-Line 9.2 & 0.03 & 2.04 & 18.93 & 8.32 & 26.72 & 0.84 & 28.98 & 1.91 \\
\hline G8527 $\times$ SREB-15C & 0.31 & 27.32 & 34.93 & 4.65 & 9.12 & 0.26 & 24.64 & 1.57 \\
\hline G8527 × UG5 & 0.03 & 3.65 & 15.26 & 6.98 & 30.82 & 0.88 & 25.98 & 1.24 \\
\hline S- Line $13.2 \mathrm{~A} \times \mathrm{SREB}-15 \mathrm{C}$ & 0.09 & 9.25 & 37.93 & 10.32 & 21.7 & 0.58 & 41.31 & 2.09 \\
\hline S-Line $13.2 \mathrm{~A}$ & 0.12 & 15.19 & 42.59 & 10.98 & 32.18 & 0.9 & 35.31 & 2.89 \\
\hline S-Line $13.2 \mathrm{~A} \times 1 \mathrm{~N}$ & 0.04 & 4.74 & 17.26 & 1.32 & 21.27 & 0.49 & 51.98 & 0.24 \\
\hline S-Line $13.2 \mathrm{~A} \times 3 \mathrm{~N}$ & 0.11 & 26.43 & 57.26 & 5.32 & 10.06 & 0.26 & 39.64 & 1.81 \\
\hline S-Line $13.2 \mathrm{~A} \times 4 \mathrm{~N}$ & -0.07 & -11.66 & 29.06 & 3.66 & -0.47 & -0.15 & 45.14 & 0.9 \\
\hline S-Line 13.2A × G7955 & 0.09 & 15.41 & 47.26 & 9.65 & 19.98 & 0.6 & 36.98 & 2.47 \\
\hline S-Line $13.2 \mathrm{~A} \times \mathrm{G} 8527$ & 0.05 & 7.83 & 16.26 & 4.32 & 34.14 & 0.97 & 42.98 & 1.35 \\
\hline S-Line $13.2 \mathrm{~A} \times \mathrm{UG} 5$ & 0.04 & 5.74 & 23.93 & 1.98 & 9.79 & 0.23 & 29.64 & 0.69 \\
\hline S-Line 9.2 & 0.04 & 6.09 & 17.59 & 1.98 & 9.78 & 0.29 & 23.31 & 0.84 \\
\hline
\end{tabular}




\begin{tabular}{|c|c|c|c|c|c|c|c|c|}
\hline S-Line $9.2 \times 1 \mathrm{~N}$ & 0.03 & 6.92 & 35.93 & 3.32 & 9.77 & 0.23 & 42.64 & 0.91 \\
\hline S-Line $9.2 \times 3 \mathrm{~N}$ & 0.3 & 25.88 & 12.59 & 0.32 & 1.67 & 0.04 & 15.31 & 0 \\
\hline S-Line $9.2 \times 4 \mathrm{~N}$ & 0.02 & 4.93 & 26.59 & 3.65 & 13.44 & 0.33 & 42.64 & 1.25 \\
\hline S-Line $9.2 \times$ G7955 & 0.03 & 5.03 & 48.93 & 4.32 & 9.41 & 0.25 & 40.31 & 1.38 \\
\hline S-Line $9.2 \times$ G8527 & 0.07 & 11.97 & 34.26 & 9.32 & 32.79 & 0.85 & 37.98 & 2.52 \\
\hline S-Line $9.2 \times$ S-Line $13.2 \mathrm{~A}$ & 0.01 & 3.49 & 26.93 & 1.32 & 4.62 & 0.12 & 35.64 & 0.42 \\
\hline S-Line $9.2 \times$ SREB-15C & 0.11 & 18.91 & 47.26 & 8.32 & 17.75 & 0.49 & 36.64 & 2.45 \\
\hline S-Line $9.2 \times$ UG 5 & 0.05 & 9.59 & 18.26 & 3.65 & 17.62 & 0.44 & 26.64 & 1.06 \\
\hline SREB-15C & 0.07 & 10.3 & 27.59 & 10.32 & 58.33 & 1.47 & 37.98 & 2.68 \\
\hline SREB- $15 \mathrm{C} \times 1 \mathrm{~N}$ & 0.06 & 10.47 & 22.26 & 7.32 & 30.23 & 0.88 & 22.98 & 1.96 \\
\hline SREB- $15 \mathrm{C} \times 3 \mathrm{~N}$ & 0.04 & 5.31 & 16.93 & 0.32 & 1.34 & 0.04 & 14.98 & 0 \\
\hline SREB- $15 \mathrm{C} \times 4 \mathrm{~N}$ & 0.04 & 8.27 & 42.59 & 3.98 & 9.92 & 0.25 & 27.31 & 1.21 \\
\hline SREB-15C $\times$ G7955 & 0.13 & 16.37 & 34.93 & 7.98 & 20.37 & 0.57 & 23.64 & 1.68 \\
\hline SREB-15C $\times$ G8527 & 0.06 & 8.21 & 21.59 & 8.65 & 38.21 & 1.3 & 29.98 & 2.64 \\
\hline SREB-15C $\times$ S-Line 9.2 & 0.05 & 6.09 & 24.26 & 0.32 & 3.72 & 0.08 & 16.64 & 0 \\
\hline SREB-15C $\times$ UG 5 & 0.09 & 10.3 & 41.59 & 11.32 & 31.97 & 0.89 & 40.64 & 2.47 \\
\hline SREB-I5C $\times$ S-Line $13.2 \mathrm{~A}$ & 0.18 & 18.83 & 54.93 & 13.32 & 20.94 & 0.59 & 36.31 & 2.73 \\
\hline UG 5 & 0.36 & 28.07 & 28.93 & 64.98 & 1827 & 65.23 & 37.64 & 4.99 \\
\hline UG $5 \times 1 \mathrm{~N}$ & 0.24 & 15.59 & 51.59 & 6.65 & 32.88 & 0.93 & 34.64 & 2.14 \\
\hline UG $5 \times 3 \mathrm{~N}$ & 0.37 & 31.91 & 30.26 & 10.32 & 27.24 & 0.89 & 32.64 & 2.48 \\
\hline UG $5 \times 4 \mathrm{~N}$ & 0.27 & 21.98 & 23.59 & 12.32 & 66.5 & 1.97 & 33.64 & 2.45 \\
\hline UG $5 \times$ G7955 & 0.35 & 22.78 & 9.59 & 1.65 & 16.63 & 0.44 & 32.98 & 0.67 \\
\hline UG $5 \times$ G8527 & 0.64 & 48.66 & 60.59 & 16.65 & 45.78 & 1.27 & 35.31 & 3.37 \\
\hline UG $5 \times$ S-Line $13.2 \mathrm{~A}$ & 0.34 & 25.25 & 11.78 & 0.52 & -6.26 & -0.25 & 23.39 & 0.13 \\
\hline UG $5 \times$ S-Line 9.2 & 0.48 & 35.43 & 44.59 & 13.98 & 38.23 & 0.67 & 30.64 & 2.77 \\
\hline$L S D$ & 0.287 & 25.7 & 42.98 & 15.45 & 491.4 & 21.3 & 18.56 & 2.7 \\
\hline
\end{tabular}

Note. swl $=$ Seed weight loss, swl\% $=$ Percent seed weight loss, Eggs $=$ Number of eggs, $\mathrm{ABE}=$ Adult bruchid emergence, $\% \mathrm{IE}=$ Percent adult insect emergence, $\mathrm{MDP}=$ Median Development Period, GI $=$ Growth index, $\mathrm{DSI}=$ Dobie susceptibility index, $1 \mathrm{~N}=$ Maksoy $1 \mathrm{~N}, 2 \mathrm{~N}=$ Maksoy $2 \mathrm{~N}, 3 \mathrm{~N}=$ Maksoy $3 \mathrm{~N}, 4 \mathrm{~N}=$ Maksoy $4 \mathrm{~N}$.

Results of the mean squares from a full diallel analysis are presented in Table 4. Crosses showed significant differences for trait swl $(\mathrm{p}<0.01)$, ABE $(\mathrm{p}<0.001)$ and DSI $(\mathrm{p}<0.001)$. General combining abilities (GCA) were significantly different for traits swl $(\mathrm{p}<0.01)$, ABE $(\mathrm{P}<0.001)$ and DSI $(\mathrm{P}<0.001)$. Specific combining abilities (SCA) were significantly different for trait ABE $(p<0.001)$ and DSI $(p<0.05)$ but was not significantly different for swl. Significant differences were observed in reciprocals for parameter swl $(\mathrm{P}<0.01)$ and $\mathrm{ABE}(\mathrm{P}<$ $0.05)$ but were not significantly different for DSI. The results showed that there were maternal effect differences for swl $(\mathrm{P}<0.01)$, ABE $(\mathrm{P}<0.01)$ and DSI $(\mathrm{P}<0.05)$. Narrow sense heritability for swl was $0.12, \mathrm{ABE}(0.17)$ and for DSI it was 0.11. Broad sense heritability for swl was 0.12, for ABE was 0.55 and 0.19 for DSI. Phenotypic variance for swl was 0.04; ABE was 215.58 and 3.54 for DSI. Seed weight loss had the highest Baker's ratio of unity $(1)$ followed by DSI $(B R=0.59)$ and the least was reported in $\mathrm{ABE}(\mathrm{BR}=0.32)$. 
Table 4. Mean squares for Callosobruchus chinensis susceptibility parameters in soybean

\begin{tabular}{lllll}
\hline Source of Variation & df & swl & ABE & DSI \\
\hline Rep & 2 & $0.03^{\mathrm{ns}}$ & $179.00^{\mathrm{ns}}$ & $9.23^{*}$ \\
Cross & 80 & $0.05^{* *}$ & $426.20^{* * *}$ & $4.88^{* * *}$ \\
GCA & 8 & $0.16^{* *}$ & $1100.39^{* * *}$ & $13.17^{* * *}$ \\
SCA & 36 & $0.02^{\mathrm{ns}}$ & $574.49^{* * *}$ & $4.33^{*}$ \\
Reciprocal & 36 & $0.05^{* *}$ & $133.50^{*}$ & $3.78^{\text {ns }}$ \\
$\quad$ Maternal & 8 & $0.13^{* *}$ & $316.60^{* *}$ & $6.90^{*}$ \\
$\quad$ No Maternal & 28 & $0.03^{\mathrm{ns}}$ & $81.19^{\mathrm{ns}}$ & $2.87^{\mathrm{ns}}$ \\
Residuals & 158 & 0.03 & 88.35 & 2.70 \\
GCA component & & 0.002 & 18.74 & 0.19 \\
SCA component & & 0.00 & 81.02 & 0.27 \\
Maternal component & & 0.002 & 4.36 & 0.08 \\
Non Maternal component & & 0.001 & 0.00 & 0.03 \\
Phenotypic Variance & & 0.04 & 215.58 & 3.54 \\
Narrow Heritability & & 0.12 & 0.17 & 0.11 \\
Broad Heritability & & 0.12 & 0.55 & 0.19 \\
Baker's ratio & & 1.00 & 0.32 & 0.59 \\
\hline
\end{tabular}

Note. df. $=$ degrees of freedom, $\mathrm{swl}=$ Seed weight loss, $\mathrm{ABE}=$ Adult bruchid emergrnce, $\mathrm{DSI}=$ Dobie Susceptibility Index, $\mathrm{ns}=$ not significant, $* * *=\mathrm{P}<0.001, * *=\mathrm{P}<0.01, *=\mathrm{P}<0.05$.

GCA effects for $C$. chinensis susceptibility parameters are presented in Table 5. Genotype Maksoy $3 \mathrm{~N}$ had positive significant GCA for swl $(\mathrm{P}<0.05)$, ABE $(\mathrm{P}<0.001)$, and DSI $(\mathrm{P}<0.05)$. Genotypes G8527, G7955 and Maksoy $1 \mathrm{~N}$ showed no significant GCA for all the parameters. Genotype Maksoy $4 \mathrm{~N}$ had positive significant GCA for ABE $(\mathrm{P}<0.001)$ and DSI $(\mathrm{P}<0.01)$. Maksoy $4 \mathrm{~N}$ had the highest GCA $\left(0.879^{* *}\right)$ for DSI and ABE $\left(6.590^{* * *}\right)$. S-Line 13.2A had significant negative GCAs for swl $(\mathrm{P}<0.05)$ and ABE $(\mathrm{P}<0.01)$ but negative non significant GCA for DSI. S-Line 13.2A had the least GCA for swl $\left(-0.049^{*}\right)$. S-Line 9.2 had significant negative GCA for DSI $(\mathrm{P}<0.01)$ and ABE $(\mathrm{P}<0.01)$ but a non significant negative GCA for swl. S-line 9.2 had the least GCA for DSI $\left(-0.69^{* *}\right)$, and ABE $\left(-5.19^{* *}\right)$. SREB-15C had negative non significant GCAs for swl and DSI but significant for $\mathrm{ABE}(\mathrm{P}<0.01)$.

Parent UG 5 had positive significant GCA for swl $(\mathrm{P}<0.001)$ and ABE $(\mathrm{P}<0.01)$ but non significant GCA for DSI. Furthermore, UG 5 presented the highest GCA $\left(0.11^{* * *}\right)$.

The estimates of parents' maternal effects are presented in Table 5. The genotypes Maksoy 4N and UG 5 had significant positive effects $(\mathrm{P}<0.01)$ for swl while only genotype AVRDC G7955 had significant positive effect $(\mathrm{P}<0.05)$ for DSI. Genotypes Maksoy $3 \mathrm{~N}$ and AVRDC G7955 had significant positive effect $(\mathrm{P}<0.05)$ while S-Line 13.2A had significant negative effect for ABE.

The estimates of SCA for the crosses are presented in Table 6. The SCA effects results showed that cross UG $\times$ SREB-15C had significant $(\mathrm{P}<0.01)$ negative SCA effect for swl. Crosses SREB-15C $\times$ Maksoy $3 \mathrm{~N}$, SREB-15C $\times$ Maksoy $4 \mathrm{~N}$, and UG $\times$ S-Line 13.2A had significant $(\mathrm{P}<0.05)$ negative SCA effects for DSI. The crosses AVRDC G8527 $\times$ AVRDC G7955, SREB-15C $\times$ AVRDC G7955 and SREB-15C $\times$ S-Line 13.2A had significant $(\mathrm{P}<0.05)$ positive effect for ABE while crosses AVRDC G7955 $\times$ Maksoy $3 \mathrm{~N}$, Maksoy $4 \mathrm{~N} \times$ Maksoy 3N, SREB-15C $\times$ Maksoy 3N, SREB-15C $\times$ Maksoy 4N, UG x AVRDC G7955, UG $\times$ Maksoy 3N and UG $5 \times$ S-Line 13.2A had significant $(\mathrm{P}<0.01)$ negative SCA effect for ABE. 
Table 5. GCA and maternal effects for Callosobruchus chinensis susceptibility parameters

\begin{tabular}{|c|c|c|c|c|c|c|}
\hline Parent & swl & Rank & DSI & Rank & ABE & Rank \\
\hline \multicolumn{7}{|l|}{ GCA effects } \\
\hline Maksoy $1 \mathrm{~N}$ & $-0.04^{\mathrm{ns}}$ & 7 & $-0.21^{\mathrm{ns}}$ & 6 & $-2.41^{\mathrm{ns}}$ & 6 \\
\hline Maksoy 3N & $0.06^{*}$ & 2 & $0.61^{*}$ & 2 & $6.57^{* * *}$ & 2 \\
\hline Maksoy 4N & $0.04^{\mathrm{ns}}$ & 3 & $0.88^{* *}$ & 1 & $6.59^{* * *}$ & 1 \\
\hline G7955 & $-0.02^{\mathrm{ns}}$ & 5 & $0.11^{\mathrm{ns}}$ & 3 & $-0.36^{\mathrm{ns}}$ & 4 \\
\hline G8527 & $-0.01^{\mathrm{ns}}$ & 4 & $0.04^{\mathrm{ns}}$ & 4 & $-2.04^{\mathrm{ns}}$ & 5 \\
\hline S-Line $13.2 \mathrm{~A}$ & $-0.05^{*}$ & 9 & $-0.38^{\mathrm{ns}}$ & 8 & $-3.51^{* *}$ & 8 \\
\hline S-Line 9.2 & $-0.04^{\mathrm{ns}}$ & 6 & $-0.69^{* *}$ & 9 & $-5.19^{* *}$ & 9 \\
\hline SREB-15C & $-0.04^{\mathrm{ns}}$ & 8 & $-0.35^{\mathrm{ns}}$ & 7 & $-3.50^{* *}$ & 7 \\
\hline UG 5 & $0.11^{* * *}$ & 1 & $0.01^{\mathrm{ns}}$ & 5 & $3.85^{*}$ & 3 \\
\hline \multicolumn{7}{|l|}{ Maternal effect } \\
\hline Maksoy $1 \mathrm{~N}$ & $-0.03^{\mathrm{ns}}$ & 7 & $0.38^{\mathrm{ns}}$ & 3 & $2.37^{\mathrm{ns}}$ & 3 \\
\hline Maksoy 3N & $0.01^{\mathrm{ns}}$ & 3 & $0.39^{\mathrm{ns}}$ & 2 & $2.94^{*}$ & 2 \\
\hline Maksoy 4N & $0.07^{* *}$ & 2 & $0.18^{\mathrm{ns}}$ & 4 & $1.41^{\mathrm{ns}}$ & 4 \\
\hline G7955 & $-0.01^{\mathrm{ns}}$ & 4 & $0.46^{*}$ & 1 & $3.05^{*}$ & 1 \\
\hline G8527 & $-0.04^{\mathrm{ns}}$ & 8 & $-0.34^{\mathrm{ns}}$ & 8 & $-1.70^{\mathrm{ns}}$ & 7 \\
\hline S-Line $13.2 \mathrm{~A}$ & $-0.04^{\mathrm{ns}}$ & 9 & $-0.46^{\mathrm{ns}}$ & 9 & $-2.94^{*}$ & 9 \\
\hline S-Line 9.2 & $-0.03^{\mathrm{ns}}$ & 6 & $-0.33^{\mathrm{ns}}$ & 7 & $-2.53^{\mathrm{ns}}$ & 8 \\
\hline SREB-15C & $-0.02^{\mathrm{ns}}$ & 5 & $-0.17^{\mathrm{ns}}$ & 6 & $-1.17^{\mathrm{ns}}$ & 5 \\
\hline UG 5 & $0.09^{* *}$ & 1 & $-0.12^{\mathrm{ns}}$ & 5 & $-1.45^{\mathrm{ns}}$ & 6 \\
\hline
\end{tabular}

Note. $\mathrm{swl}=$ Seed weight loss, DSI $=$ Dobie Susceptibility Index, $\mathrm{ABE}=$ Adult Bruchid Emergence, $\mathrm{ns}=$ not significant, $* * *=\mathrm{P}<0.001,{ }^{* *}=\mathrm{P}<0.01, *=\mathrm{P}<0.05$. 
Table 6. SCA effects for Callosobruchus chinensis susceptibility parameters on soybean $\mathrm{F}_{2}$ generations

\begin{tabular}{|c|c|c|c|c|c|c|}
\hline Cross & DSI & Rank & swl & Rank & $\mathbf{A B E}$ & Rank \\
\hline G7955 × Maksoy $1 \mathrm{~N}$ & $-0.58^{\mathrm{ns}}$ & 27 & $0.01^{\mathrm{ns}}$ & 15 & $-1.64^{\mathrm{ns}}$ & 19 \\
\hline G7955 $\times$ Maksoy $3 \mathrm{~N}$ & $-0.64^{\mathrm{ns}}$ & 29 & $-0.03^{\mathrm{ns}}$ & 26 & $-7.12^{*}$ & 30 \\
\hline G7955 $\times$ Maksoy $4 \mathrm{~N}$ & $-0.05^{\mathrm{ns}}$ & 17 & $0.06^{\mathrm{ns}}$ & 8 & $1.53^{\mathrm{ns}}$ & 8 \\
\hline $\mathrm{G} 8527 \times \mathrm{G} 7955$ & $0.86^{\mathrm{ns}}$ & 2 & $-0.01^{\mathrm{ns}}$ & 19 & $7.32^{*}$ & 1 \\
\hline G8527 $\times$ Maksoy $1 \mathrm{~N}$ & $-0.89^{\mathrm{ns}}$ & 32 & $-0.07^{\mathrm{ns}}$ & 34 & $-2.30^{\mathrm{ns}}$ & 22 \\
\hline G8527 $\times$ Maksoy $3 \mathrm{~N}$ & $-0.25^{\mathrm{ns}}$ & 21 & $0.07^{\mathrm{ns}}$ & 7 & $-3.11^{\mathrm{ns}}$ & 25 \\
\hline G8527 $\times$ Maksoy $4 \mathrm{~N}$ & $0.18^{\mathrm{ns}}$ & 13 & $-0.03^{\mathrm{ns}}$ & 27 & $-2.63^{\mathrm{ns}}$ & 24 \\
\hline Maksoy $3 \mathrm{~N} \times$ Maksoy $1 \mathrm{~N}$ & $0.58^{\mathrm{ns}}$ & 6 & $0.01^{\mathrm{ns}}$ & 16 & $-0.74^{\mathrm{ns}}$ & 14 \\
\hline Maksoy $4 \mathrm{~N} \times$ Maksoy $1 \mathrm{~N}$ & $-0.34^{\mathrm{ns}}$ & 23 & $0.09^{\mathrm{ns}}$ & 3 & $-5.26^{\mathrm{ns}}$ & 28 \\
\hline Maksoy $4 \mathrm{~N} \times$ Maksoy $3 \mathrm{~N}$ & $-0.24^{\mathrm{ns}}$ & 19 & $-0.03^{\mathrm{ns}}$ & 28 & $-7.24^{*}$ & 31 \\
\hline S-Line $13.2 \mathrm{~A} \times \mathrm{G} 7955$ & $0.86^{\mathrm{ns}}$ & 3 & $0.04^{\mathrm{ns}}$ & 10 & $4.46^{\mathrm{ns}}$ & 4 \\
\hline S-Line $13.2 \mathrm{~A} \times \mathrm{G} 8527$ & $-0.43^{\mathrm{ns}}$ & 26 & $-0.04^{\mathrm{ns}}$ & 30 & $-1.36^{\mathrm{ns}}$ & 17 \\
\hline S-Line $13.2 \mathrm{~A} \times$ Maksoy $1 \mathrm{~N}$ & $-0.38^{\mathrm{ns}}$ & 25 & $0.00^{\mathrm{ns}}$ & 17 & $0.01^{\mathrm{ns}}$ & 13 \\
\hline S-Line $13.2 \mathrm{~A} \times$ Maksoy $3 \mathrm{~N}$ & $0.23^{\mathrm{ns}}$ & 11 & $-0.03^{\mathrm{ns}}$ & 25 & $-1.31^{\mathrm{ns}}$ & 16 \\
\hline S-Line $13.2 \mathrm{~A} \times$ Maksoy $4 \mathrm{~N}$ & $-0.24^{\mathrm{ns}}$ & 20 & $-0.07^{\mathrm{ns}}$ & 35 & $-2.00^{\mathrm{ns}}$ & 21 \\
\hline S-Line $9.2 \times$ G7955 & $-0.35^{\mathrm{ns}}$ & 24 & $-0.06^{\mathrm{ns}}$ & 33 & $-1.24^{\mathrm{ns}}$ & 15 \\
\hline S-Line $9.2 \times \mathrm{G} 8527$ & $0.64^{\mathrm{ns}}$ & 5 & $-0.04^{\mathrm{ns}}$ & 29 & $4.32^{\mathrm{ns}}$ & 5 \\
\hline S-Line $9.2 \times$ Maksoy $1 \mathrm{~N}$ & $0.45^{\mathrm{ns}}$ & 7 & $-0.01^{\mathrm{ns}}$ & 20 & $1.86^{\mathrm{ns}}$ & 7 \\
\hline S-Line $9.2 \times$ Maksoy $3 \mathrm{~N}$ & $-0.26^{\mathrm{ns}}$ & 22 & $0.08^{\mathrm{ns}}$ & 5 & $-3.79^{\mathrm{ns}}$ & 26 \\
\hline S-Line $9.2 \times$ Maksoy $4 \mathrm{~N}$ & $0.02^{\mathrm{ns}}$ & 16 & $0.04^{\mathrm{ns}}$ & 11 & $0.86^{\mathrm{ns}}$ & 11 \\
\hline S-Line $9.2 \times$ S-Line $13.2 \mathrm{~A}$ & $-0.94^{\mathrm{ns}}$ & 33 & $-0.05^{\mathrm{ns}}$ & 31 & $-2.38^{\mathrm{ns}}$ & 23 \\
\hline SREB-15C $\times$ G7955 & $0.71^{\mathrm{ns}}$ & 4 & $0.01^{\mathrm{ns}}$ & 14 & $6.95^{*}$ & 3 \\
\hline SREB-15C $\times$ G8527 & $0.19^{\mathrm{ns}}$ & 12 & $0.10^{\mathrm{ns}}$ & 2 & $0.46^{\mathrm{ns}}$ & 12 \\
\hline SREB-15C $\times$ Maksoy $1 \mathrm{~N}$ & $0.39^{\mathrm{ns}}$ & 9 & $0.01^{\mathrm{ns}}$ & 13 & $4.00^{\mathrm{ns}}$ & 6 \\
\hline SREB-15C $\times$ Maksoy $3 \mathrm{~N}$ & $-1.36^{*}$ & 34 & $-0.06^{\mathrm{ns}}$ & 32 & $-10.48^{* *}$ & 34 \\
\hline SREB-15C $\times$ Maksoy $4 \mathrm{~N}$ & $-1.39^{*}$ & 35 & $-0.02^{\mathrm{ns}}$ & 22 & $-8.50^{* *}$ & 32 \\
\hline SREB-15C $\times$ S-Line $13.2 \mathrm{~A}$ & $0.91^{\mathrm{ns}}$ & 1 & $0.08^{\mathrm{ns}}$ & 4 & $7.10^{*}$ & 2 \\
\hline SREB-15C $\times$ S-Line 9.2 & $0.04^{\mathrm{ns}}$ & 14 & $0.02^{\mathrm{ns}}$ & 12 & $1.29^{\mathrm{ns}}$ & 9 \\
\hline UG $5 \times$ G7955 & $-0.88^{\mathrm{ns}}$ & 31 & $-0.02^{\mathrm{ns}}$ & 23 & $-10.56^{* *}$ & 35 \\
\hline UG $5 \times$ G8527 & $0.04^{\mathrm{ns}}$ & 15 & $0.104^{\mathrm{ns}}$ & 1 & $-1.72^{\mathrm{ns}}$ & 20 \\
\hline UG $5 \times$ Maksoy $1 \mathrm{~N}$ & $0.43^{\mathrm{ns}}$ & 8 & $-0.03^{\mathrm{ns}}$ & 24 & $0.99^{\mathrm{ns}}$ & 10 \\
\hline UG $5 \times$ Maksoy $3 \mathrm{~N}$ & $-0.59^{\mathrm{ns}}$ & 28 & $0.07^{\mathrm{ns}}$ & 6 & $-10.33^{* *}$ & 33 \\
\hline UG $5 \times$ Maksoy $4 \mathrm{~N}$ & $-0.05^{\mathrm{ns}}$ & 18 & $-0.02^{\mathrm{ns}}$ & 21 & $-5.18^{\mathrm{ns}}$ & 27 \\
\hline UG $5 \times \mathrm{S}$-Line $13.2 \mathrm{~A}$ & $-1.44^{*}$ & 36 & $-0.01^{\mathrm{ns}}$ & 18 & $-10.79^{* *}$ & 36 \\
\hline UG $5 \times$ S-Line 9.2 & $0.38^{\mathrm{ns}}$ & 10 & $0.05^{\mathrm{ns}}$ & 9 & $-1.56^{\mathrm{ns}}$ & 18 \\
\hline UG $5 \times$ SREB- $15 \mathrm{C}$ & $-0.64^{\mathrm{ns}}$ & 30 & $-0.16^{* *}$ & 36 & $-6.42^{\mathrm{ns}}$ & 29 \\
\hline
\end{tabular}

Note. swl $=$ Seed weight loss, DSI $=$ Dobie Susceptibility Index, $\mathrm{ABE}=$ Adult Bruchid Emergence, $\mathrm{ns}=$ not significant, $* * * \mathrm{P}<0.001, * *=\mathrm{P}<0.01, *=\mathrm{P}<0.05$.

\section{Discussions}

To develop an efficient and successful resistance breeding programme, understanding the genes controlling resistance is fundamental. Studying the mode of inheritance and the number of genes controlling resistance is very important because choice of breeding method depends on this (Keneni et al., 2011). For example where bruchid resistance is influenced by cytoplasmic gene effects, backcross methods would be more applicable. However, information from the literature on bruchid resistance inheritance studies in soybean is scanty. The genetic control of resistance to storage insect pests may range from monogenic to polygenic (Dent, 2000; Singh, 2009). Mostly additive and dominant genes may govern storage insect pest resistance in many legumes but a few cases of cytoplasmic gene effect have also been reported (Singh, 2009; Keneni et al., 2011).

In this study nine parents were crossed in a full diallel to determine the gene action and inheritance of resistance to $C$. chinensis. From the present study genotypes were significantly different for swl, ABE and DSI. GCA and maternal effects were significant $(\mathrm{P}<0.05)$ for swl, DSI and $\mathrm{ABE}$ from $\mathrm{F}_{2}$ soybean seeds. Susceptibility parameters ABE and DSI recorded significant SCA. Genotypes S-Line 9.2 and S-Line 13.2A recorded negative significant GCA effects for at least two of the susceptibility parameters.

Genotypes showed variability in response to bruchid infestation for swl, ABE and DSI (Table 2) implying that crosses and parents exhibited varying levels of resistance thereby providing an opportunity for crop improvement work on soybean. Wayne et al. (2004) reported that genetic variation could be due to either cis- or trans-acting factors at transcript level. A gene might contain a mutation in its cis-regulatory region, and/or the 
amount or activity of trans-regulating factors controlling its expression might vary (Murtadha et al., 2018). The cis- and trans-acting elements are the two complimentary regulatory components in the transcriptional machinery of eukaryotes. The gene expression differences between parental alleles usually observed as phenotypic variations is a result from cis- and/or trans regulatory changes. Further these results basically implied that the swl, ABE and DSI could further be used for screening genotypes in the genetic analyses. Parameters Eggs, MDP and GI did not show variability among $\mathrm{F}_{2}$ genotypes, therefore could not be used in the genetic analyses. These findings are in agreement with Kananji (2007) who reported variability in susceptibility parameters in dry beans.

The results indicated a decrease in DSI with hybridization except for crosses with female parent Maksoy $1 \mathrm{~N}$ and S-Line 9.2 (Table 3), thus implying that hybridization can increase resistance probably by reducing the number of $\mathrm{ABE}$ and increasing the MDP. Derera et al. (2014) also reported similar findings. Similar results were observed in the reciprocal crossing which showed no significant influence on DSI.

According to Hallauer et al. (1988) the average performance of a parent in a hybrid combination is termed GCA, whilst the deviation of the performance of a hybrid from the expectation based, on the average GCA effects of the lines that produced the hybrid is termed the SCA. GCA is equivalent to the breeding value and estimated additive genetic variation (Wayne et al., 2004). Significant GCAs were observed among parents indicating differences in performance of genotypes as parents in hybrid combinations (Mwije et al., 2014). Further more significant differences in the three parameters of swl, ABE and DSI indicated that genes controlling soybean resistance to $C$. chinensis lack dominance (Abakemal et al., 2011) and their gene action is additive, therefore the expression of the offspring were intermediate (Table 4). This suggests that selection of parents to generate resistant crosses and developing resistant pure line cultivars is possible (Mulbah et al., 2015). These results further indicated that selection would be effective and it could be used to fix resistance in cultivars (Fasahat et al., 2016). However SCA mean squares for ABE and DSI were significant indicating that resistance to C. chinensis was also influenced by non additive gene effects signifying the presence of a locus or loci with dominance variation (Fasahat et al., 2016). Presence of significant SCA indicates that a complex type of inheritance to resistance of $C$. chinensis may be involved in some parents (Hakizimana et al., 2004). Similar conclusions were drawn by Kananji (2007) in beans and Somta et al. (2007) in mungbeans. The presence of both additive and non additive gene actions might indicate transgressive segregation in soybean (Machado et al., 2009). Wayne et al., (2004) reported that if parental alleles were purely additive, then an $F_{2}$ genotype would deviate from the population mean by the sum of the GCAs of its parents and due to environmental or error effects. Any additional deviation from the population mean would be attributable either to dominance, i.e., intralocus interactions, of alleles or to epistasis, i.e., interlocus interactions (Cai et al., 2012).

This study showed that maternal effects were significant (Table 4), indicating that resistance to C. chinensis depended on the genotype of maternal parent used in hybridization (Vaiserman et al., 2013). In fact all the significant effects of reciprocal crosses were attributed to maternal effects since non-maternal effects were not significant for all the traits implying that seed resistance was controlled by chemicals synthesized by the female parent and transported to the cotyledon and embryo of the seed (Somta et al., 2007). This finding is in agreement with Fernandez and Talekar (1990), and Somta et al. (2007), who concluded that resistance to bruchids in mungbean was controlled by the maternal genotype. Maternal effects indicate that the traits had mitochondrial mode of inheritance pattern implying that when selecting parents for hybridization in soybean for bruchid resistance breeders should give female parent priority (Cai et al., 2012). The significant difference between reciprocal crosses indicated that non- chromosomal maternal effects could have contributed to a heterotic response (Mendes et al., 2015), and presence of the interaction of the genes with nuclear factors or the expression of extranuclear genes (Cai et al., 2012).

Seed weight loss and DSI recorded high Baker's ratio of 1 and 0.59 respectively (Table 4) and this implied preponderance of additive gene action. The unity baker's ratio for swl indicated total influence of additive gene effects (Baker 1978). The Baker's ratio of unity or closer to unity indicated greater predictability of progeny performance based on the GCA alone (Cai et al., 2012) and better transmission of trait to the progenies (Murtadha, et al. 2018). In soybean a self pollinating crop this implied that when non additive genes are lost after some generations, it would practically be possible to fix genes controlling these traits. The breeding procedures to be adopted for these characters include pure line selection, mass selection, progeny selection, hybridization and selection and heterosis breeding (Choudhary et al., 2004). The bakers'ratio for ABE was 0.32, indicating that non-additive gene effects were more important than additive gene effects in controlling the inheritance of this trait in the germplasm evaluated. Cai et al. (2012) reported that performance of hybrids for traits with low Baker's ratio couldn't be predicted from GCA but from exploiting of the SCA. Therefore, only crossing the two 
parents with the lowest ABE-GCA effects cannot simply produce the best C. chinensis resistant progeny (Hakizimana et al., 2004) as such breeding procedure for this character should be performed using heterosis breeding method (Choudhary et al., 2004).

Estimation of the components of variance for a quantitative trait allows one to evaluate both the degree to which genes influence the trait and the trait's underlying genetic architecture (Abney et al., 2001). The narrow sense heritability results in Table 4, indicated that additive gene action was present but not high as such early generation selection would not be very effective. High narrow sense heritability of closer to 1 or $100 \%$ indicates that early generation selection would be effective (Hansen, 1989). Adult bruchid emergence had a high broad sense heritability which was an indication of high dominance genetic contribution towards phenotypic variance. Seed weight loss had broad and narrow sense heritabilities equal indicating no significant dominance effect (Bahmankar et al., 2014).

The GCA effects results for the parents (Table 5) clearly showed that genotype Maksoy 1N, G7955 and G8725 had no significant GCA for any of the traits suggesting that these genotypes influence on the studied traits did not depend on the partner combining with them. Parent UG 5 and Maksoy $3 \mathrm{~N}$ contributed highest to swl while SREBC-15C and S-Line 13.2A contributed the least to swl (Table 5) suggesting the best donors for developing reduced swl varieties would be SREB-15C and S-Line 13.2A. Genotype UG 5 would generate the populations with highest mean swl while the population with least swl would be generated by S-Line 13.2A (Dias et al., 2017).

Parent Maksoy 4N and Maksoy 3N contributed highly to DSI while S-Line 9.2 contributed the least to DSI indicating that Maksoy $4 \mathrm{~N}$ and Maksoy $3 \mathrm{~N}$ would increase susceptibility of genotypes in hybridization (Fernandez \& Talekar, 1990) while S-Line 9.2 was best combiner for bruchid resistance amongst the parents. Parent S-Line 9.2 contributed the least to adult bruchid emergence while parent Maksoy $4 \mathrm{~N}$ and Maksoy $3 \mathrm{~N}$ contributed the highest to ABE indicating that S-Line 9.2 was best combiner for reduction of ABE. The results from this study indicated that it was not easy to get one donor genotype for all traits of interest (Murtadha et al., 2018). The results suggested that to improve soybean resistance to bruchids, it was imperative that genotypes with negative GCAs should be selected (Maphosa, 2013).

Out of the 9 genotypes used in the study, 6 had negative GCA effects and only three (Maksoy 3N, Maksoy 4N and UG 5) had positive GCA effects. Negative general combining abilities are preferred for pest and disease resistance because they are based on the scale where highest values are associated with more pest infestation (Fasahat et al., 2016).

Significant SCA effects (Table 6) were observed in 11 out of 36 of crosses, indicating the presence of nonadditive gene effects. Crosses with significant negative SCA effects such as SREB-15C $\times$ S-Line 13.2A, SREB-15C $\times$ Maksoy 3N, AVRDC G7955 $\times$ Maksoy 3N just to mention a few would be very beneficial in the development of $C$. chinensis resistant varieties. The significant and positive SCA effects presented by cross AVRDC G8527 (Resistant) $\times$ AVRDC G7955 (Resistant) could not be explained very well but Kananji (2007) reported that this happens due to quantitative inheritance of genes, however Symphorien (2018) reported that accidental resistance break down could happen when two or more resistant genotypes are combined. A number of studies (Abakemal et al., 2011; Nagarajan et al., 2017) observed the same results but gave no explanation for it, which therefore warrants more studies on the factors creating such a situation.

Significant and negative SCA effects were observed for the combination UG $5 \times 3 \mathrm{~N}(\mathrm{~S} \times \mathrm{S})$ suggesting that resistance of these progenies was higher or lower than would be expected from the average resistance of their respective parents thereby implying that resistant genotypes could be produced from susceptible parents due to transgressive segregation or inter and intra-locus gene interactions (Hakizimana et al., 2004).

\section{Conclusions and Recommendations}

The study identified SREB-15C, S-Line 9.2 and S-Line 13.2A as useful parents in breeding for resistance to $C$. chinensis based on general combining abilities. The study established that additive and non-additive gene effects governed the gene expression in soybean resistance to C. chinensis. The presence of maternal effects signified the importance of ensuring that parental lines with most desired traits are always made females during hybridization. The best progenies from crosses with negative GCAs should further be screened and advanced for the release of resistant varieties.

This study generated knowledge on the genetic inheritance and start up material for the breeding programme. In a nutshell, there are heritable quantitative traits controlling bruchid resistance which can be exploited in the soybean breeding program. However, there is need to test more germplasm lines including the wild relatives of 
soybean and more hybrid development by screening segregating $F_{2}$ soybean populations. This will enable to validate the consistency of the gene action. Our results relied on phenotypic data, we suggest follow up studies to map and identify quantitative trait loci associated with resistance to bruchids. The identification of QTL is key in marker assisted breeding which would bypass the challenges and lengthy procedures associated with conventional breeding.

\section{Acknowledgements}

We are grateful for the financial support from Malawi Government through APPSA Project, Intra ACP-Mobility Scheme (CSAA) and Carnegie Cooperation (New York) through RUFORUM-Grant RU/2016/INTRA $\mathrm{ACP} / \mathrm{RG} / 013$. We acknowledge the Soybean Breeding and Seed Systems program of Makerere University for providing the germplasm. The authors appreciate the technical support from Mr. George Yiga, Mr. Paul Kabayi and Ms. Naziwa of MUARIK.

\section{References}

Abakemal, D., Zelleke, H., Kanuajia, K. R., \& Wegary, D. (2011). Combining ability in maize lines for agronomic triats and resistance to weevil (Sitophilus zeamais Motschulky). Ethiopian Journal of Crop Science, 2(1), 37-48.

Abebe, F., Tefera, T., Mugo, S., Beyene, Y., \& Vidal, S. (2009). Resistance of maize varieties to the maize weevil Sitophilus zeamais (Motsch.) (Coleoptera: Curculionidae). African Journal of Biotechnology, 8(21), 5937-5943. https://doi.org/10.5897/AJB09.821

Abney, M., Mcpeek, M. S., \& Ober, C. (2001). Broad and narrow heritabilities of quantitative traits in a founder population. The American Society of Human Genetics., 68(5), 1302-1307. https://doi.org/10.1086/320112

Adjadi, O., Singh, B. B., \& Singh, S. R. (1985). Inheritance of bruchid resistance in cowpea. American Society of Agronomy, 25(5), 740-742. https://doi.org/10.2135/cropsci1985.0011183X002500050005x

Amusa, O. D., Ogunkanmi, L. A., Adetunbi, J. A., Akinyosoye, S. T., Bolarinwa, K. A., \& Ogundipe, O. T. (2014). Assessment of bruchid (Callosobruchus maculatus) tolerance of some elite cowpea (Vigna unguiculata) varieties. Journal of Agriculture and Sustainability, 6(2), 164-178.

Bahmankar, M., Raij, M. R., Seloki, A. R., \& Shirkool, K. (2014). Assessment of broad sense heritability and genetic advance in safflower assessment of broad sense heritability and genetic advance in safflower. International Journal of Biosciences, 4(8), 131-135. https://doi.org/10.12692/ijb/4.8.131-135

Cai, Q. S., Wang, L. L., Yao, W. H., Zhang, Y. D., Liu, L., Yu, L. J., \& Fan, X. M. (2012). Diallel analysis of photosynthetic traits in maize. Crop Science, 52(2), 551-559. https://doi.org/10.2135/cropsci2011.06.0333

Choudhary, M., Kumawat, K. R., \& Kumawat, R. (2004). Diallel analysis and its applications in plant breeding. Biotech Articles. Jobner-303329, Jaipur, India: Department of Plant Breeding and Genetics, S.K.N. Agriculture University. Retrieved from http://www.biotecharticles.com.privacy.php

Dabholkar, A. R. (1999). Elements of biometrical genetics (2nd ed.). New Delhi, India: Concept Publishing Company.

Dent, D. (2000). Insect pest management (2nd ed.). CABI Bioscience. https://doi.org/10.1079/97808519934 09.0000

Derera, J., Pixley, K. V., Giga, D. P., \& Makanda, I. (2014). Resistance of maize to the maize weevil: III. Grain weight loss assessment and implications for breeding. Journal of Stored Products Research, 59(10), 24-35. https://doi.org/10.1016/j.jspr.2014.04.004

Dias, F. T. C., Bertini, F. C. H., \& Francisco, R. F. (2017). Genetic effects and potential parents in cowpea. Crop Breeding and Applied Biotechnology, 16(4). https://doi.org/10.1590/1984-70332016v16n4a47

Dongre, T. K., Pawar, S. E., Thakare, R. G., \& Harwalkar, M. R. (1996). Identification of resistant sources to cowpea weevil. Journal of Stored Products Research, 32(3), 201-204. https://doi.org/10.1016/S0022-474X (96)00028-8

Fasahat, P., Rajabi, A., Rad, J. M., \& Derera, J. (2016). Principles and utilization of combining ability in plant breeding. Biometrics \& Biostatistics International Journal, 4(1), 1-24. https://doi.org/10.15406/bbij.2016. 04.00085

Fernandez, G. C., \& Talekar, N. S. (1990). Genetics and breeding for bruchid resistance in asiatic Vigna species. In K. Fujii, A. M. R. Gatehouse, C. D. Johnson, R. Mitchel \& T. Yoshida (Eds.), Bruchids and Legumes: 
Economics, Ecology and Coevolution (Series Entomologica, Vol. 46). Springer, Dordrecht. https://doi.org/ 10.1007/978-94-009-2005-7_23

Hakizimana, F., Ibrahim, A. M. H., Langham, M. A. C., Haley, S. D., \& Rudd, J. C. (2004). Diallel analysis of wheat streak mosaic virus resistance in winter wheat. Crop Science, 44, 89-92. https://doi.org/10.1007/ s10681-004-2490-y

Hallauer, A. R., Carena, M. J., \& Miranda-Filho, J. (1988). Quantitative genetics in maize breeding (2nd ed.). New York: Springer.

Hansen, M. (1989). Genetic variation and inheritance of tolerance to clubroot \{Plasmodiophora brassicae Wor.) and other quantitative characters in cabbage (Brassica oleracea L.). Hereditas, 110(1), 13-22. https://doi.org/10.1111/j.1601-5223.1989.tb00412.x

Harding, S., \& Payne, R. (2012). Multivariate analysis: A Guide to Multivariate Analysis in GenStat ${ }^{\circledR}$ (pp. 27-32). VSN International, 5 The Waterhouse, Waterhouse Street, Hemel Hempstead, Hertfordshire HP1 $1 \mathrm{ES}, \mathrm{UK}$.

Kananji, G. (2007). A study of bruchid resistance and its inheritance in Malawian dry bean germplasm. (Unpublished doctoral dissertation, University of KwaZulu-Natal, Pretoria, Republic of South Africa).

Keneni, G., Bekele, E., Getu, E., Imtiaz, M., Damte, T., Mulatu, B., \& Dagne, K. (2011). Breeding food legumes for resistance to storage insect pests: Potential and limitations. Sustainability, 3(9), 1399-1415. https://doi.org/10.3390/su3091399

Machado, J., Souza, J., Ramalho, M. A., \& Lima, J. L. (2009). Stability of combining ability effects in maize hybrids. Scientia Agricola: Genetics and Plant Breeding, 66(4).

Maphosa, M. (2013). Enhancing genetic resistance to soybean rust disease (Unpublished doctoral dissertation, Makerere University, Kampala, Uganda).

Mendes, M. H. S., Pereira, C. H., \& de Souza, J. C. (2015). Análise dialélica de híbridos de milho para características agronômicas e bromatológicas da forragem. Acta Scientiarum. Agronomy, 37(2), $141-146$. https://doi.org/10.4025/actasciagron.v37i2.19329

Msiska, U. M., Odong, T. L., Hailay, M., Miesho, B., Kyamanywa, S., Rubahaiyo, P. R., \& Tukamuhabwa, P. (2018). Resistance of Uganda soybean germplasm to adzuki bean bruchid. African Crop Science Journal, 26(3). https://doi.org/http://dx.doi.org/10.4314/acsj.v26i3.2

Mulbah, Q. S., Shimelis, H. A., \& Laing, M. D. (2015). Combining ability and gene action of three components of horizontal resistance against rice blast. Euphytica, 203(2). https://doi.org/10.1007/s10681-015-1522-0

Murtadha, M. A., Ariyo, O. J., \& Alghamdi, S. S. (2018). Analysis of combining ability over environments in diallel crosses of maize (Zea mays). Journal of the Saudi Society of Agricultural Sciences, 17(1), 69-78. https://doi.org/10.1016/j.jssas.2016.01.004

Mwije, A., Mukasa, S. B., Gibson, P., \& Kyamanywa, S. (2014). Heritability analysis of putative drought adaptation traits in sweetpotato. African Crop Science Journal, 22(1), 79-87.

Nagarajan, D., Kalaimagal, T., \& Murugan, E. (2017). Combining ability analysis for yield component and biochemical traits in soybean [Glycine $\max (\mathrm{L}$.$) Merrill]. International Journal of Current Microbiology$ and Applied Sciences, 6(11), 2894-2901.

Nahdy, S. M. (1995). Biotic and abiotic factors influencing the biology and distribution of common storage pests of pigeon pea (Unpublished doctoral dissertation, University of Reading, UK).

Qazi, M. A. (2007). Development and monthly percent damage of Callosobruchus chinensis L. Pakistan Journal of Agricultural Research, 20(3-4).

Radha, R., \& Susheela, P. (2014). Studies on the life history and ovipositional preference of Callosobruchus maculatus reared on different pulses. Research Journal of Animal, Veterinary and Fishery Sciences, 2(6), $1-5$.

Seram, D., Mohan, S., Kennedy, J. S., \& Senthil, N. (2016). Development and damage assessment of the storage beetle, Callosobruchus maculatus under normal and controlled conditions. In S. Navarro, D. S. Jayas, \& K. Alagusundaram (Eds.), Proceedings ot the $10^{\text {th }}$ International Conference on controlled atmosphere and fumigation in stored products (CAF2016) (pp. 25-31). CAF Permanent Committee Secretariat, Winnipeg, Canada. 
Singh, B. D. (2009). Plant breeding: Principles and methods (4th ed.). New Delhi-Ludhiana: Kalyaki Publishers. https://doi.org/10.1111/j.1439-0523.2008.01576.x

Somta, P., Ammaranan, C., Ooi, P. A. C., \& Srinives, P. (2007). Inheritance of seed resistance to bruchids in cultivated mungbean (Vigna radiata, L. Wilczek). Euphytica. https://doi.org/10.1007/s10681-006-9299-9

Symphorien, A., Karungi, J., Badji, A., Kassim, S., Paul, G., Edema, R., ... Rubaihayo, P. R. (2018). Inheritance of cowpea resistance to flower thrips in Uganda germplasm inheritance of cowpea resistance to flower thrips in Uganda germplasm. Journal of Plant Breeding and Crop Science, 10(1), 21-32. https://doi.org/10.5897/JPBCS2017.0698

Vaiserman, A. M., Zabuga, O. G., Kolyada, A. K., Pisaruk, A. V., \& Kozeretska, I. A. (2013). Reciprocal cross differences in Drosophila melanogaster longevity: An evidence for non-genomic effects in heterosis phenomenon? Biogerontology, 14(2), 153-163. https://doi.org/10.1007/s10522-013-9419-6

Wayne, M. L., Pan, Y. J., Nuzhdin, S. V., \& McIntyre, L. M. (2004). Additivity and trans-acting effects on gene expression in male Drosophila simulans. Genetics, 168(3), 1413-1420. https://doi.org/10.1534/genetics.104. 030973

Wijenayake, D. U. S., \& Karunaratne, M. M. S. C. (1999). Ovipositional preference and development of the cowpea beetle Callosobruchus chinensis on different stored pulses. Vidyodaya J. of Sci., 1, 8135-8147.

Zunjare, R., Hossain, F., Muthusamy, V., Jha, S. K., Kumar, P., Sekhar, J. C., ... Gupta, H. S. (2015). Genetics of resistance to stored grain weevil (Sitophilus oryzae L.) in maize. Cogent Food \& Agriculture, 1(1), 1-9. https://doi.org/10.1080/23311932.2015.1075934

\section{Copyrights}

Copyright for this article is retained by the author(s), with first publication rights granted to the journal.

This is an open-access article distributed under the terms and conditions of the Creative Commons Attribution license (http://creativecommons.org/licenses/by/4.0/). 